\title{
Möglichkeiten zur Verbesserung der Medikamentenadhärenz im Kindes- und Jugendalter
}

Weitreichende Fortschritte in der Medizin eröffnen Kindern und Jugendlichen mit chronischen Gesundheitsstörungen neue Chancen zur Verbesserung ihrer Symptomlast, zur Reduktion von Komplikationen und Langzeitfolgen sowie zur Steigerung der sozialen Teilhabe. Der Erfolg der Therapien hängt jedoch wesentlich davon ab, ob es den Familien gelingt, diese in ihrem Alltag konsequent umzusetzen.

Die Messung der Adhärenz, also des Ausmaßes, in dem das Verhalten eines Patienten mit den Empfehlungen des Behandlers übereinstimmt, ist schwierig. Abhängig von der Definition und den Erfassungsmethoden kommen Studien zu sehr heterogenen Ergebnissen (vgl. DiMatteo 2004). Die Weltgesundheitsorganisation (WHO) schätzt jedoch, dass es über verschiedene pädiatrische Erkrankungen nur durchschnittlich 50\% der Patienten gelingt, eine gute Adhärenz zu erzielen (Sabaté 2003). Wenn man davon ausgeht, dass die verordneten Maßnahmen für ein optimales Therapieergebnis notwendig sind, bekommen damit die Hälfte der Patienten keine optimale Therapie.

Sah man früher die fehlende Motivation des Patienten und seiner Familie als Hauptgrund für Non-Adhärenz, geht man heute von einem komplexen Zusammenspiel vieler verschiedener Faktoren aus (Sabaté 2003; - Tab. 1). Personen, die das Therapieregime nicht einhalten, sind selten einfach unmotiviert, sondern häufig ambivalent. D. h. sie kennen gute Gründe, die für die Therapie sprechen (z. B. Verringern von Krankheitssymptomen, Vermeiden von Krankheitsfolgen), aber auch viele Gründe gegen die dauerhafte Einnahme der Medikamente (z.B. fehlende Zuversicht hinsichtlich des Therapieerfolgs, Angst vor Nebenwirkungen, Aufwand im Zusammenhang mit der Einnahme, Einschränkung der Lebensqualität). Insbesondere bei komplexen Therapien fehlen vielen $\mathrm{Pa}$ tienten auch das Wissen und die Fertigkeiten, die Therapie adäquat umzusetzen (z. B. inkorrekte Berechnung der Medikamentendosis, fehlerhafte Injektion von Medikamenten), oder die Therapieumsetzung scheitert an Barrieren im Alltag (z. B.
Widerstand des Kindes gegen die Therapie, Vergessen der Therapie durch konkurrierende Aktivitäten, Weigerung von Kindertagesstätten, Medikamente zu verabreichen).

Auch Faktoren, die nicht im Einflussbereich des Patienten liegen, sondern beim Behandlungsteam oder dem Gesundheitssystem, können die Therapietreue beeinflussen. Fehlende Kostenübernahme, Zuzahlungen, geringe Dichte qualifizierter Behandlungseinrichtungen und lange Wartezeiten erschweren die adäquate Therapie.

Die Non-Adhärenz hat demnach vielfältige Ursachen. Entsprechend vielfältig und auf die individuellen Ursachen abgestimmt müssen daher die Maßnahmen zur Verbesserung der Adhärenz sein. Osterberg \&
Blaschke (2005) gliedern die Ansätze in vier Kategorien:

- Bessere Information und Schulung von Patienten

- Vereinfachung der Einnahmeschemata

- Leichterer Zugang zu klinischen Versorgungsangeboten und kürzere Wartezeiten

- Verbesserte Kommunikation zwischen Arzt und Patient

Maßnahmen, die sich auf die rein kognitive Vermittlung von Wissen konzentrieren, greifen in der Regel zu kurz. Als erfolgreicher haben sich verhaltensbasierte und mehrere Komponenten umfassende Maßnahmen erwiesen, die auf die Stärkung des Selbstmanagements der Familien abzielen (Kahana et al. 2008). Dazu gehören neben am Empowerment orientierten Patientenschulungen der altersangemesse-

- Tab. 1 Prädiktoren-Cluster der Adhärenz (angelehnt an WHO, Sabaté 2003).

1. Patientenbezogene Faktoren

- Kognitive Leistungsfähigkeit

- Emotionale Stabilität, psychische Gesundheit

- Akzeptanz der Krankheit, Verständnis für die Notwendigkeit der Behandlung

- Laienätiologie

- Vertrauen in die Therapie

- Krankheitsrelevante Kenntnisse und Fertigkeiten

2. Krankheitsbezogene Faktoren

- Dauer der Erkrankung: chronisch vs. akut

- Schwere der Symptome und gesundheitlichen Einschränkungen

- Komorbiditäten

- Progression

3. Behandlungsbezogene Faktoren

- Komplexität der Therapiepläne

- Anzahl der täglichen Medikamenteneinnahmen

- Therapiedauer

- Spür-/sichtbare Wirkung, Latenz bis Wirkeintritt

- Nebenwirkungen sowie Angst vor Nebenwirkungen

- Beeinträchtigung anderer Lebensbereiche und der Lebensqualität

4. Faktoren im Zusammenhang mit dem Gesundheitssystem und der Versorgung

- Vertrauensverhältnis zwischen Arzt und Patient, Kommunikation

- Verfügbarkeit qualifizierter Angebote, Wartezeiten

- Finanzierung von Schulung und Beratung

- Einheitlichkeit von Empfehlungen

5. Faktoren im Zusammenhang mit der sozioökonomischen Situation

- Alter

- Bildungsniveau

- Finanzielle Situation

- Familiäre/soziale Unterstützung

- Zusätzliche Belastungsfaktoren (z. B. alleinerziehend, Migrationsbiografie) 
ne Einbezug in die Therapie, das Einräumen von Wahlmöglichkeiten, das Vereinbaren von Zielen, Token-Systeme, bei denen das Kind nach jeder erfolgreichen Medikamenteneinnahme belohnt wird, und generelle Verstärkung, um die Selbstwirksamkeitserwartung zu stärken.

Abhängig vom Zeitpunkt in der Therapie können folgende einfach in den klinischen Alltag integrierbare Maßnahmen helfen:

\section{Diagnosestellung}

Wenn Familien erstmalig mit einer Diagnose oder neuen Therapie konfrontiert werden, sind sie häufig überfordert angesichts der Fülle von Informationen. Insbesondere starke Emotionen wie Angst und Verzweiflung erschweren das Verstehen von Informationen. In diesen Situationen kann eine wiederholte, auf den Wissensbedarf der Familie abgestimmte Information über die verschiedenen Therapieoptionen Erleichterung bringen. In mehreren Gesprächen sollten realistische Ergebniserwartungen vermittelt und Befürchtungen hinsichtlich der Einnahme und möglicher negativer Auswirkungen offen thematisiert werden. Gesprächstechniken wie das Motivational Interviewing (Miller \& Rollnick 2009) können beim Aufdecken und Bearbeiten von Ambivalenzen hilfreich sein.

\section{Planung der Therapiedurchfüh- rung und Schulung der Familien}

Bei der konkreten Planung der Therapie sollte die Einfachheit des Therapieschemas im Vordergrund stehen (u.a. wenige Tagesdosen, Verwendung von Kombinationspräparaten), um den Therapieaufwand und mögliche Fehler zu minimieren. Dabei müssen die individuelle Situation und die Bedürfnisse der Familie berücksichtigt werden.

Bei komplexeren Therapien brauchen die Familien zudem die notwendigen Kenntnisse und Fertigkeiten, um die Therapie korrekt umzusetzen. Dazu gehört z. B. das praktische Training zur Nutzung eines Pens oder Inhalators und zur Festlegung der Dosierung, ebenso wie die Information, welche Probleme auftreten können und was dann zu tun ist. Mögliche Barrieren, die eine Therapieumsetzung im Alltag erschweren könnten, sollten offen diskutiert werden. Gemeinsam sollte überlegt werden, was der Familie helfen kann, die Barrieren zu reduzieren.

Bei Typ-1-Diabetes, Asthma bronchiale, Neurodermitis und Adipositas, die ein intensives Selbstmanagement der Familien erfordern, sind derartige Schulungen integraler Bestandteil der Langzeittherapie (Ernst et al. 2013). Sie gehen über die reine Instruktion zum Medikamentengebrauch hinaus und vermitteln alltagsrelevante Kenntnisse zur Therapie und greifen auch emotionale und praktische Probleme der Krankheitsbewältigung im Familienalltag auf.

\section{Verstetigung der Therapie im Alltag}

Viele Probleme bei der Therapieumsetzung fallen erst bei der Anwendung im Alltag auf. Eine engmaschige Betreuung durch das Behandlungsteam in der Initialphase ist daher notwendig. Aber auch im Langzeitverlauf sollte ein regelmäßiges Monitoring der Adhärenz stattfinden. Das Auftreten von Nebenwirkungen oder Problemen bei der Therapieumsetzung sollte dabei offen erfragt, und Erfolge sollten aufgezeigt werden. Langfristig ist es hilfreich, wenn die Therapie Gewohnheitsstärke bekommt, indem sie z. B. an andere Gewohnheiten wie das abendliche Zähneputzen gekoppelt wird.

Ziel all dieser Maßnahmen ist es laut Lange (2012), „... die notwendige Therapie so im Alltag zur Routine werden zu lassen, dass alle Schritte zuverlässig bedacht und durchgeführt werden, ohne dass es zu erheblichen Beeinträchtigungen anderer Le- bensziele und Aufgaben kommt." (S. 61). Dies wird nur im regelmäßigen Austausch zwischen Familie und Behandlungsteam gelingen.

\section{Interessenkonflikte}

Es bestehen keine Interessenkonflikte.

Autor

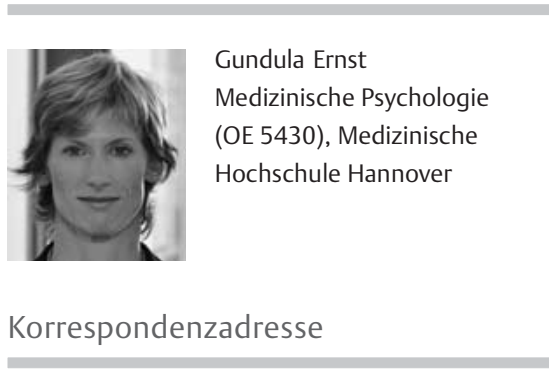

Dr. Gundula Ernst

Medizinische Psychologie (OE 5430)

Medizinische Hochschule Hannover

Carl-Neuberg-Straße 1

30625 Hannover

Ernst.gundula@mh-hannover.de

Literatur

[1] DiMatteo MR. Variations in patients' adherence to medical recommendations: a quantitative review of 50 years of research. Med Care 2004; 42: 200-209

[2] Ernst G, Szczepanski R, Lange K. Patientenschulung in der Kinder- und Jugendmedizin - Bestandsaufnahme deutschsprachiger Konzepte und Bedarfsanalyse. Prävention und Rehabilitation 2013; 25: 18-24

[3] Kahana S, Drotar D, Frazier T. Meta-analysis of psychological interventions to promote adherence to treatment in pediatric chronic health conditions. J Pediatr Psychol 2008; 33: $590-611$

[4] Lange K. Therapieadhärenz bei Typ-1-Diabetes: Herausforderungen, Barrieren und Chancen. Diabetologe 2012; 8: 55-66

[5] Miller WR, Rollnick S. Motivierende Gesprächsführung. Freiburg: Lambertus; 2009

[6] Osterberg L, Blaschke T. Adherence to medication. N Engl J Med 2005; 353: 487-497

[7] Sabaté E. Adherence to long-term Therapies: Evidence for Action. Geneva: World Health Organization; 2003 